\title{
Guest Editorial: Tenth International Conference on Computability, Complexity and Randomness (CCR 2015)
}

\author{
Andrew Lewis-Pye ${ }^{1}$ - Wolfgang Merkle ${ }^{2}$
}

Published online: 19 September 2017

(C) Springer Science+Business Media, LLC 2017

We are pleased to present this special issue of Theory of Computing Systems, which is devoted to emerging themes in the study of algorithmic complexity. The articles appearing in this volume were produced, upon the invitation of the editors, by speakers at the Tenth International Conference on Computability, Complexity and Randomness (CCR 2015). The conference took place in Heidelberg as part of the focus semester on Computability and Randomness, which was organised and supported by the Mathematics Center Heidelberg.

In "Semiautomatic structures" by Jain, Khoussainov, Stephan, Teng and Zou, the authors introduce a generalisation of the class of automatic structures, for which one only requires that the derived relations and functions are automatic when all but one input are fixed with constant values. While automatic structures of various types have played an important role in group theory and logic, the notion is too restrictive to be widely applicable. Semiautomatic structures are introduced and analysed as a response to the natural question as to whether there are general ways to utilise finite automata for the presentation of non-automatic structures such as the natural numbers with multiplication and equality.

This article is part of the Topical Collection on Special Issue on Computability, Complexity and Randomness (CCR 2015)

Andrew Lewis-Pye

thelewisboy123@gmail.com

1 London School of Economics Department of Mathematics, London School of Economics and Political Science, Columbia House, Houghton St, London WC2A 2AE, UK

2 Institute of Computer Science, Heidelberg University, Im Neuenheimer Feld 294, 69120, Heidelberg, Germany 
In "Exact constructive and computable dimensions" by Ludwig Staiger, the author provides a systematic development of exact constructive dimension, which quantitatively refines constructive dimension just as the classical theory of exact Hausdorff dimension refines the usual Hausdorff dimension.

In "Conditional probabilities and van Lambalgen's Theorem revisited", by Bauwens, Shen and Takahashi, the authors provide an engaging survey of a line of research in algorithmic probability theory which is concerned with such questions as to how to define conditional probability distributions relative to random sequences, and the extent to which van Lambalgen's Theorem can be generalised within the resulting theory.

In "A generalised characterization of algorithmic probability" by Sterkenburg, the author approaches foundational issues and questions in statistics via the study of universal semimeasures. Universal semimeasures are a central object of study in algorithmic randomness, and can be used to give a concrete definition of what it means to be random. While the standard approach to creating these semimeasures uses the probability of outputting a string from a universal machine when the input is chosen at random using the uniform measure, the author establishes that under mild conditions the uniformity requirement is not necessary, and that, in fact, any computable continuous measure will suffice.

In "Layerwise computability and image randomness" by Bienvenu, Hoyrup and Shen, the authors consider the notion of layerwise computability, and explain its importance in clarifying how algorithmic randomness interacts with intuitive and fundamental principles of randomness conservation.

In "On the uniform computational content of computability theory" by Brattka, Hendtlass and Kreuzer, the authors show how the Weihrauch lattice can be used to classify the uniform computational content of computability theoretic properties and theorems in one common setting. The paper describes a precise sense in which many theorems in computability theory, including all theorems which claim the existence of some Turing degree, have very little uniform content and argue that this may yield an explanation for why results in computability typically have very few direct consequences in other disciplines such as analysis.

In "On the complexity of automatic complexity" by Bjørn Kjos-Hanssen, the author generalises the notion of automatic complexity for individual words to give a notion of automatic complexity $A(E)$ for an equivalence relation $E$ on a finite set of words $S$. Amongst other results, it is established that the problem of determining whether $A(E)$ equals the number $|E|$ of equivalence classes of $E$ is NP-complete.

In "Short lists with short programs from programs of functions and strings" by Vereshchagin, the author gives a precise negative solution to the question as to whether there is a total algorithm which produces a short list containing a short program for a given function, when provided with an index for a program which computes that function.

Collectively these paper provide a small selection of the diverse range of issues currently being investigated in algorithmic complexity. We hope they will provide inspiration for future research in this exciting field. 